\title{
Miniature In-Phase Wilkinson Power Divider with Pair of Parallel Transmission-Lines for Application in Wireless Microwave Systems
}

\author{
Farzad Khajeh-Khalili ${ }^{1}$, M. Amin Honarvar ${ }^{2 *}$, Bal S. Virdee ${ }^{3}$ \\ ${ }^{1}$ Department of Electrical Engineering, Najafabad Branch, Islamic Azad University, Najafabad, Iran \\ e-mail: khalili.farzad@gmail.com \\ ${ }^{2}$ Department of Electrical Engineering, Najafabad Branch, Islamic Azad University, Najafabad, Iran \\ *Corresponding author: e-mail: amin.honarvar@pel.iaun.ac.ir \\ ${ }^{3}$ Faculty of Life Sciences and Computing, Center for Communications Technology, London Metropolitan \\ University, London, United Kingdom \\ e-mail: b.virdee@londonmet.ac.uk
}

\begin{abstract}
A novel miniature Wilkinson power divider (WPD) design is presented. This is achieved by substituting the quarter-wavelength transmission-lines constituting the WPD with an equivalent pair of parallel transmission-lines (PPTLs) that effectively reduce the circuit size of the WPD by $43 \%$ compared with a conventional design whose ground-plane is defected. Particle swarm optimization (PSO) technique is used to achieve size reduction. Meandering the transmission-lines further reduce the WPD size by $22 \%$. The proposed WPD has overall dimensions of $9.38 \times 11.51 \mathrm{~mm}^{2}$ or $0.11 \lambda_{g} \times 0.14 \lambda_{g}$, which is compatible with the requirements imposed by portable personal wireless systems. Coupling between the parallel transmission-lines is shown to extend the operational bandwidth of the power divider to $3.2 \mathrm{GHz}(0.8-4 \mathrm{GHz})$ for a return-loss better than $10 \mathrm{~dB}$.
\end{abstract}

Index Terms - Wilkinson power divider (WPD), pair of parallel transmission-lines (PPTLs) miniaturization, particle swarm optimization (PSO), defected ground structure (DGS). 


\section{INTRODUCTION}

The Wilkinson power divider (WPD) is an essential component for power division or combination, which is necessary in wireless communication systems due to the isolation between its output ports [1]. These devices also find applications in mixers, balanced amplifiers, and antenna arrays. The main challenge facing conventional WPDs is its large circuit size, especially at low microwave frequencies, and limited bandwidth due to the use of a pair of quarter-wavelength transmission-lines in its design [2]. With the advent of multiband wireless communication systems, a lot of studies have been done over the recent years for designing multi-band or wideband WPD [3-10]. In [3], a dual-band WPD is proposed using a two-section transmission-line transformer with its output ports connected through a parallel $R L C$ circuit for improving its isolation at the two bands. At its two operating frequencies however the power selectivity of the device is low. This deficiency can improved by adding a resonant open or short-circuited stub at the junction of the two output ports. In [5], a novel dual-band WPD is presented using the model of $\pi$-transmission-line in place of the common quarter-wavelength transmission-line. In [6], isolation between two output ports is improved by adding a complex isolation component between two 90 transmission-lines at an arbitrary phase angle from the input terminal; however, the design is band limited. Threesection transmission-line transformer is used to design a tri-band WPD in [7]. In [8] a quadband WPD consists of three transmission-lines and two loaded shorted-stubs. Although the above designs have good isolation between their output ports, however their dimensions are relatively large for today's communication systems. In [10], we have presented a novel wideband triple lines WPD using PSO technique which operates from 0.5 to $4.5 \mathrm{GHz}$ for application in several wireless communication standards.

More recently, the design of WPDs have become more compact [11-17]. For example, in [11] the WPD is proposed using two section asymmetrical T-structures to achieve a significant size reduction. The asymmetrical T-structure is realized by two series highimpedance lines with unequal electrical lengths and a shunt low-impedance line. Although these designs are compact in size however their bandwidth is limited.

In this paper, a novel WPD is presented that operates over a wideband and is highly compact in size. The power divider is composed of a pair of parallel transmission-lines (PPTLs) whose ground-plane is defected. Particle swarm optimization (PSO) algorithm is used to achieve size reduction for predetermined conditions such as width of lines and reflection-coefficient. The proposed structure of the WPD is shown in Figure 1. Measured results confirm the power divider operates over a frequency range of $0.8-4 \mathrm{GHz}$, which 
includes the following bands: GSM 850 (824-894 MHz), GSM 900 (890-960 MHz), GPS (1565-1585 MHz), GSM 1800 (1710-1885 MHz), GSM 1900 (1850-1990 MHz), DCS (1.71-1.85 GHz), PCS (1.85-1.99 GHz), UMTS (1.92-2.17 GHz), WLAN+Blutooth (2.4, 2.48 GHz), WiMAX (2.5-2.69 GHz), WiMAX (3.3-3.5 GHz), and WLAN (3.65-3.7 GHz). It should be noted that the authors of this paper, in [10], also presented a very small and high performance wideband WPD. But to the authors' knowledge, the proposed wideband WPD in this article is one of the smallest structures presented to date.

\section{EQIVALENT MODEL REPRESENTING A QUARTER-WAVELENGTH TRANSMISSION-LINE}

The size of a quarter-wavelength transmission-line of given impedance $Z$ is reduced by replacing it with a pair of parallel transmission-lines of impedance $Z_{1}$ and $Z_{2}$ of electrical

lengths $\theta_{1}$ and $\theta_{2}$, respectively, as illustrated in Figure 2. The proposed reduction technique provides a wideband structure as discussed in [10].

The admittance matrix for a transmission-line with characteristic admittance $Y$ and electrical length $\theta$ is represented by [2]:

$\left[\begin{array}{cc}Y_{11} & Y_{12} \\ Y & Y \\ 21 & { }_{22}\end{array}\right]=\left[\begin{array}{ll}-j Y \cot \theta & j Y \csc \theta \\ j Y \csc \theta & -j Y \cot \theta\end{array}\right]$,

Therefore, the admittance matrix of a quarter-wavelength transmission-line with electrical length $\theta=90^{\circ}$ is given by:

$\left[\begin{array}{cc}Y_{11} & Y_{12} \\ Y & Y \\ 21 & 22\end{array}\right]_{\lambda / 4}=\left[\begin{array}{ll}0 & j Y \\ j Y & 0\end{array}\right]$,

The admittance matrix for a pair of transmission-lines is given by:

$$
\begin{aligned}
& {\left[\begin{array}{cc}
Y_{11} & Y_{12} \\
Y & Y \\
21 & 22
\end{array}\right\rfloor_{\text {Line } 1}=\left[\begin{array}{cc}
-j Y_{1} \cot \theta_{1} & j Y_{1} \csc \theta_{1} \\
j Y \csc \theta_{1} & -j Y_{1} \cot \theta_{1} \\
1 & 1
\end{array}\right],}
\end{aligned}
$$

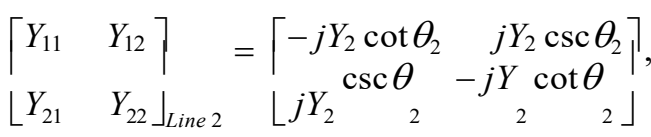

In relations (3) and (4), the electrical lengths are $\theta_{2}<90^{\circ}<\theta_{1}$. In the proposed structure in

Figure 2, the admittance matrix of the quarter-wavelength transmission-line is equivalent to the total of admittance matrix of the two parallel transmission-lines, i.e.:

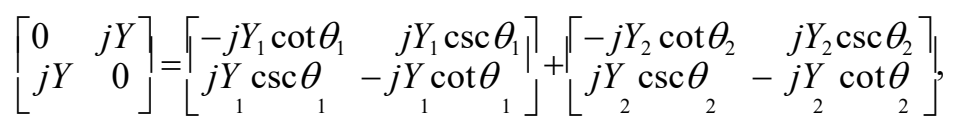

Simplification of (5) reduces to: 
$Y_{1} \cot \theta_{1}+Y_{2} \cot \theta_{2}=0$

$Y_{1} \csc \theta_{1}+Y_{2} \csc \theta_{2}=0$,

Where

$$
\begin{aligned}
& Y_{1}=Y \cdot \frac{\sin \theta_{1} \cos \theta_{2}}{\cos \theta_{2}-\cos ^{2} \theta_{1}} \\
& Y_{2}=-Y \cdot \frac{\cos \theta_{1} \sin \theta_{2}}{\cos \theta_{2}-\cos ^{2} \theta_{1}}
\end{aligned}
$$

Or

$$
\begin{gathered}
Z=Z \cdot \frac{\cos \theta_{2}-\cos \theta_{1}}{\sin \theta_{1} \cos \theta_{2}}, \\
Z_{2}=-Z \cdot \frac{\cos \theta_{2}-\cos \theta_{1}}{\cos \theta_{1} \sin \theta_{2}}
\end{gathered}
$$

Where impedance $Z=70.71 \Omega$ [2]. The four unknown parameters $\left(\theta_{1}, \theta_{2}, Z_{1}\right.$, and $\left.Z_{2}\right)$ in (10) and (11) need to be optimized in order to miniaturize the WPD design. The PSO technique is used here to provide the optimum solution. On the other hand, each pair of transmission-lines in the proposed PPTLs needs to exhibit an analogous performance as a conventional quarterwavelength transmission-line resonator. The pair of TLs in PPTLs generates a pair of resonating frequencies. Coupling between these two resonating structures is exploited here to significantly enhance the overall bandwidth of the WPD. The resonating frequencies can be controlled by adjusting lengths $\theta_{1}$ and $\theta_{2}$. The PPTL structure was designed on a RO4003 substrate with relative permittivity of 3.55 , thickness of $1.524 \mathrm{~mm}$, and $\tan \delta=0.0027$. The center frequency of the proposed structure is $2.4 \mathrm{GHz}$. If, for example, $\theta_{1}$ and $\theta_{2}$ are $78.03^{\circ}$ and $103.69^{\circ}$, respectively, the corresponding frequency response of the PPTLs is shown in Figure 3 along with the response of a conventional quarter-wavelength transmission-line. The PPTL excites two resonant frequencies at $2.2 \mathrm{GHz}$ and $2.6 \mathrm{GHz}$. The PPTL structure was simulated and optimized using CST Design Studio 2016. The results confirm a wideband WPD can be realized using PPTL with good return-loss across its passband.

\section{OPTIMIZATION OF WPD PARAMETERS}

\subsection{PSO Algorithm Process}

PSO is a powerful algorithm which lies within the swarm intelligence field introduced by Kennedy and Eberhart [18]. PSO has recently gained popularity because of its simplicity and functionality [19-21]. The technique draws inspiration from social behavior of the living animals such as birds that live together in small and large groups. The technique assumes all 
members or particles of the group are connected with one another, where they interact and interchange information with each other. These particles are spread within a function search space, where the location of each particle is calculated using the objective function. By composing information about each particles present location and the best place it has been before, a direction for movement is determined. These steps are repeated until reaching the favorite or optimized solution. PSO, just like other evolutionary algorithms, begins with an initial random population. The initial population comprises a number of $N$ particles which are randomly given an initial quantity. Each particle has a location vector and velocity vector. These particles start moving in the search space and find better locations by calculating the objective function value. Each particle needs two memories for search; a memory is allocated to store the best location present among all particles. Using the information taken from these memories, the particles decide how to move in the next stage. In each repetition of the process, all particles update their velocity and location according to the best absolute and local answers [22]. The location of each particle in population is calculated by adding the velocity of the particle to its present location based on following relationship:

$X_{j}(i)=X_{j}(i-1)+V_{j}(i)$,

Where $X_{j}(i)$ shows the location of the $j^{\text {th }}$ particle, and $V_{j}(i)$ shows the velocity, and $i$ shows iterance of the velocity variable. The velocity is calculated using the relation:

$V_{j}(i)=\theta(i) V_{j}(i-1)+c_{1} r_{1}\left[P_{\text {best }, j}-X_{j}(i-1)\right]+c_{2} r_{2}\left[G_{\text {best }}-X_{j}(i-1)\right]$,

In (13), $V_{j}(i)$ is the $i^{\text {th }}$ component of the $j^{\text {th }}$ particle velocity; $r_{1} \quad$ and $r_{2}$ are two random

numbers with uniform distribution in $(0,1) ; c_{1}$ and $c_{2}$ parameters are the individual and group learning factors based on experimental results [22]; $P_{\text {best }, j}$ and $G_{\text {best }}$ are, respectively, the best local location the particle has ever met and the best overall location that all particles have so far reached; and $\theta_{i}$ is inertia weight to control the particles velocity in laboratory repetitions. $\theta_{i}$ is given by [23]:

$\theta(i)=\theta-\left(\frac{\theta_{\text {max }}-\theta_{\text {min }}}{i_{\max }}\right) i$,

Where $\theta_{\min }$ and $\theta_{\max }$ are, respectively, the initial values and the final inertia weight; and $i_{\max }$ is the maximum iterance number in algorithm. For $\theta_{\min }$ and $\theta_{\max }$ it has been experimentally proved that if the values are as $\theta_{\min }=0.4$ and $\theta_{\max }=0.7$, the results are usually the best [10].

\subsection{PSO Optimization of the WPD}


PSO algorithm is used to optimize the electrical lengths of the PPTLs and their corresponding characteristic impedances centered at $2.4 \mathrm{GHz}$. Impedances of the microstrip transmissionlines where limited to maximum of $150 \Omega\left(Y=6.67 \times 10^{-3} \mathrm{mho}\right)$ as it was not possible to fabricate them. The PSO algorithm parameters are set to reaching the favorite miniaturization size based on practical fabrication conditions. The parameters used in the PSO algorithm are given in Table 1.

The functions to achieve the desired objectives are:

$$
\begin{aligned}
& F_{1}=\left\{\begin{array}{ll}
0 & Y_{1}>6.67 \times 10^{-3} \\
1 & \text { others }
\end{array},\right. \\
& F_{2}=\left\{\begin{array}{ll}
0 & Y_{2}>6.67 \times 10^{-3} \\
1 & \text { others }
\end{array},\right.
\end{aligned}
$$

The objective function can be defined as:

$$
F=\min \left(\sum_{i=1}^{2} F_{i}\right), \quad i=1,2 .
$$

The PSO algorithm is written in MATLAB. The code very quickly performs analysis. A sample convergence performance of the PSO algorithm can be shown in Figure 4.

According to Figure 4, in 200 iterations algorithm converges to a good approximate answer and within 82 iterations the answer can be found. Different runs were done and the sample results algorithm for the optimized electrical lengths $\theta_{1}$ and $\theta_{2}$ are shown in Table 2 .

Based on Table 2, to choose optimized $\theta_{1}$ and $\theta_{2}$, trial 86 will be chosen. Hence, in continue, these results will be used. The final results from the PSO algorithm are shown in Table 3.

\section{DESIGN AND CONFIGURATION OF THE PROPOSED WPD}

CST Microwave Studio 2016 was used to simulate the PSO Wilkinson power divider structure centered at $2.4 \mathrm{GHz}$ from the results in Section 3. The insertion-loss and return-loss response of the proposed WPD is shown in Figure 5. The parallel transmission-lines in the WPD structure, inset in Figure 5, are folded to make the device compact. However, meanderline technique was used to compensate this defect and further compress the circuit size by $22 \%$. The WPD operates over $0.8-4 \mathrm{GHz}$ for $\mathrm{S}_{11}<-10 \mathrm{~dB}$.

Further size reduction of the proposed WPD was achieved by implementing DGS in the ground-plane under the PPTLs to disturb the current distribution over it in order to increase the structures effective capacitance and inductance [24]. The DGS pattern etched on the 
ground-plane is shown in Figure 6. The effect of various DGS parameters, tabulated in Table 4 , on the power divider's $S_{11}$ response is shown in Figure 7 . The results show the center frequency of the device can be tuned from 2.4 to $1.9 \mathrm{GHz}$ by simply modifying the DGS parameter $L_{g 2}$. This parameter increases the capacitance of transmission-lines, thus effectively increasing their electrical lengths without any physical change.

DGS parameters given in case 4 of Table 4 were employed to reduce the physical size of the WPD. Figure 8 shows the structure of the PPTLs in microstrip form used in the WPD. The upper transmission-line has impedance and electrical length $Z_{1}$ and $\theta_{1}$, respectively; and the lower transmission-line has impedance and electrical length $Z_{2}$ and $\theta_{2}$, respectively. These parameters were obtained using PSO. The dimensions of these structures are (all in millimeters): $W_{1}=0.34, W_{2}=0.24, L_{1}=4, L_{2}=1, L_{3}=2.445, L_{4}=3, L_{5}=0.76, L_{6}=0.48, L_{7}$ $=1.76, L_{8}=2.02, L_{9}=0.24, L_{10}=6.68$, and $L_{11}=4.26$. So, the proposed WPD effectively reduce the circuit size of the WPD by $43 \%$ compared with a conventional design [15].

The final configurations of the proposed WPD and the conventional WPD are shown in Figures 9(a) and 9(b), respectively. It can be seen that the proposed WPD is significantly smaller than the conventional one. The $50 \Omega$ input and output ports are attached to SMA connectors. Figure 10 shows the insertion-loss, return-loss, and isolation performance of the proposed WPD and the conventional WPD at the center frequency of $2.4 \mathrm{GHz}$. According to Figure 10, the proposed WPD covers a wideband from $0.8-4 \mathrm{GHz}$ for $\mathrm{S}_{11}<-10 \mathrm{~dB}$. This is achieved by replacing the quarter-wavelength sections of transmission-lines with an equivalent PPTLs with two closely spaced resonance frequencies. This is while the bandwidth of the conventional WPD is $400 \mathrm{MHz}(1.8-3.2 \mathrm{GHz})$.

\section{EXPERIMENTAL RESULTS}

The proposed WPD was fabricated to determine the accuracy of the design and simulated results. A photograph of the fabricated WPD is shown in Figure 11. The dimensions of the power divider are $9.38 \times 11.51=107.96 \mathrm{~mm}^{2}$ or $0.11 \lambda_{g} \times 0.14 \lambda_{g}$, where $\lambda_{g}$ is the guide wavelength at the center frequency of $2.4 \mathrm{GHz}$. The measurements on the device were conducted using Agilent network analyzer HP8722ES.

Figure 12 shows the measured and simulated insertion-loss, return-loss, and isolation parameter of the device. An ideal WPD splits the input power equally between its output ports, i.e. $S_{21}=-3 \mathrm{~dB}$. The simulated and measured value of parameter $\mathrm{S}_{21}$ at the center frequency of $2.4 \mathrm{GHz}$ is $-3.06 \mathrm{~dB}$ and $-3.2 \mathrm{~dB}$, respectively. The additional loss incurred is due to conductor and dielectric substrate. The power divider has a bandwidth of $3.2 \mathrm{GHz}$ for 
$S_{11}<-10 \mathrm{~dB}$. The discrepancy between the simulated and measured results of $4.2 \%$ is attributed to the manufacturing tolerance. The isolation $\mathrm{S}_{23}$ in a WPD is achieved with a 100 $\Omega$ resistor placed between the output ports. At the center frequency the simulated isolation is $-43 \mathrm{~dB}$ and the measured isolation of $-45 \mathrm{~dB}$ is recorded at $2.2 \mathrm{GHz}$. Also, magnitude and phase difference have been shown in Figures 13(a) and 13(b), respectively. The in-band amplitude imbalance is less than $0.022 \mathrm{~dB}$. In addition, the measured phase difference is about $\pm 0.1^{\circ}$. It indicates a very good in-phase performance. Table 5 shows the proposed WPD in comparison with prior dividers. It shows the proposed wideband WPD is one of the smallest structures presented to date.

\section{CONCLUSION}

A highly compact wideband WPD is presented. This was achieved by substituting the quarter-wavelength transmission-line with an equivalent parallel transmission-line structure with DSG etched under it. The parameters of the structure were optimized using PSO technique. The simulation and measured results confirm the device exhibits excellent insertion-loss, return-loss, and isolation characteristics. The wideband device covers frequency bands of GSM 850, GSM 900, GPS, GSM 1800, GSM 1900, DCS/PCS/UMTS, WLAN, and WiMAX.

\section{References}

1. E. J. Wilkinson, -An N-way hybrid power divider,l IRE Trans. Microw. Theory Tech., vol. 8, no. 1, pp. 116-118, Jan. 1960.

2. D. M. Pozar, Microwave Engineering, 3rd ed. Hoboken, NJ: Wiley, 2005, pp. 318-323.

3. L. Wu, H. Yilmaz, A. Pascht, and M. Berroth, -A dual-frequency Wilkinson power divider: For a frequency and its first harmonic,\| IEEE Microw. Wirel. Compon. Lett., vol. 15, no. 2, pp. 107-109, Feb. 2005.

4. A. R. Moznebi and K. Afrooz, Power divider/combiner using half mode substrate integrated waveguide (HMSIW) technology with high power and high isolation,\|IETE J. Res., vol. 63, no. 4, pp. 558-564, Feb. 2017.

5. K. - K. - M. Cheng and F. - L. Wong, -A new Wilkinson power divider for dual band application,\|IEEE Microw. Wirel. Compon. Lett., vol. 17, no. 9, pp. 664-666, Sep. 2007. 
6. X. Wang, I. Sakagami, A. Mase, and M. Ichimura, -Wilkinson power divider with complex isolation component and its miniaturization, IEEE Trans. Microw. Theory Tech., vol. 62, no. 3, pp. 422-430, Jan. 2014.

7. M. Chongcheawchamnan, S. Patisang, M. Krairiksh, and I. D. Robertson, -Tri-band Wilkinson power divider using a three-section transmission-line transformer,\| IEEE Microw. Wirel. Compon. Lett., vol. 16, no. 8, pp. 452-454, Jul. 2006.

8. B. Xia, L. -S. Wu, J. Mao, and L. Yang, -A new quad-band Wilkinson power divider, J. Electromagn. Waves Appl., vol. 28, no. 13, pp. 1622-1634, Jul. 2014.

9. K. Song, M. Fan, M. Zhang, Y. Zhu, and Y. Fan, -Compact triple-band power divider integrated bandpass-filtering response using short-circuited SIRs, IEEE Trans. Compon., Packag., Manuf. Technol. vol. 7, no. 7, pp. 1144-1150, Jul. 2017.

10. F. Khajeh-Khalili and M. A. Honarvar, -A design of triple lines Wilkinson power divider for application in wireless communication systems, $\|$ J. Electromagn. Waves Appl., vol. 30, no. 16, pp. 2110-2124, Oct. 2016.

11. C. - H. Tseng and C. - H. Wu, -Compact planar Wilkinson power divider using $\pi-$ equivalent shunt-stub-based artificial transmission lines,\| Electron. Lett., vol. 46, no. 19, pp. 1327-1328, Sep. 2010.

12. A. Dadgarpour, G. Dadashzadeh, M. Naser-Moghadasi, F. Jolani, and B. S. Virdee, -PSO/FDTD optimization technique for designing UWB in-phase power divider for linear array antenna application,\|IEEE Anteanns Wirel. Propag. Lett., vol. 6, pp. 424427, May 2010.

13. U. T. Ahmed and A. M. Abbosh, -Design of wideband single-layer in-phase power divider using microstrip to slotline coupled structure,\| Microw. Opt. Technol. Lett., vol. 57, no. 4, pp. 789-791, Apr. 2015.

14. K. Sarhadi and M. Shahabadi, -Wideband substrate integrated waveguide power splitter with high isolation,\|IET Microw. Antennas Prop., vol. 4, no. 7, pp. 817-821, Jul. 2010.

15. R. Mirzavand, M. M. Honari, A. Abdipour, and G. R. Moradi, -Compact Microstrip Wilkinson power dividers with harmonic suppression and arbitrary power division ratios, IEEE Trans. Microw. Theory Tech., vol. 61, no. 1, pp. 61-68, Jan. 2013.

16. U. T. Ahmed and A. M. Abbosh, -Extremely wideband in-phase power divider using modified Wilkinson design,॥ Microw. Opt. Technol. Lett., vol. 57, no. 8, pp. 1799-1802, Aug. 2015. 
17. H. Tian, J. Gao, M. Su, and Y. Liu, -Super miniaturized Wilkinson power divider using cross-connect meander microstrip lines and conductive vias, $\|$ J. Electromagn. Waves Appl., vol. 30, no. 2, pp. 188-194, Dec. 2015.

18. J. Kennedy and R. Eberhart, -Particle swarm optimization,\| in IEEE Conference on Neural Networks, Australia, 1995, pp. 1942-1948.

19. R. Bera, D.Mandal, R. Kar, and S. P. Ghoshal, -Optimal design of single and multi-ring planar array antenna using simplex-PSO,\| IETE J. Res., Jun. 2017. doi: http://dx.doi.org/10.1080/03772063.2017.1331146.

20. M. Sharifi Sorkherizi and A. A. Kishk, -Use of group delay of sub-circuits in optimization of wideband large-scale bandpass filters and diplexers,॥ IEEE Trans. Microw. Theory Tech., vol. 65, no. 8, pp. 2893-2905, Aug. 2017.

21. B. Babakhani and S. K. Sharma, -Dual null steering and limited beam peak steering using triple-mode circular microstrip patch antenna, IEEE Trans. Antennas Propag., vol. 65, no. 8, pp. 3848-2905, Aug. 2017.

22. R. C. Eberhart and Y. Shi, -Particle swarm optimization: Developments, applications and resources,\| in Proc. IEEE Int. Conf. Evolutionary Computation, vol. 1, 2001, pp. 81-86.

23. Y. Shi and R. C. Eberhart, -A modified particle swarm optimizer, in Proc. IEEE Int. Conf. Evolutionary Computation, 1998, pp. 69-73.

24. V. Crnojević-Bengin, Advances in Multi-Band Microstrip Filters, Cambridge University Press, 2015, pp. 140-190. 
IETE Journal of Research

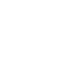

-
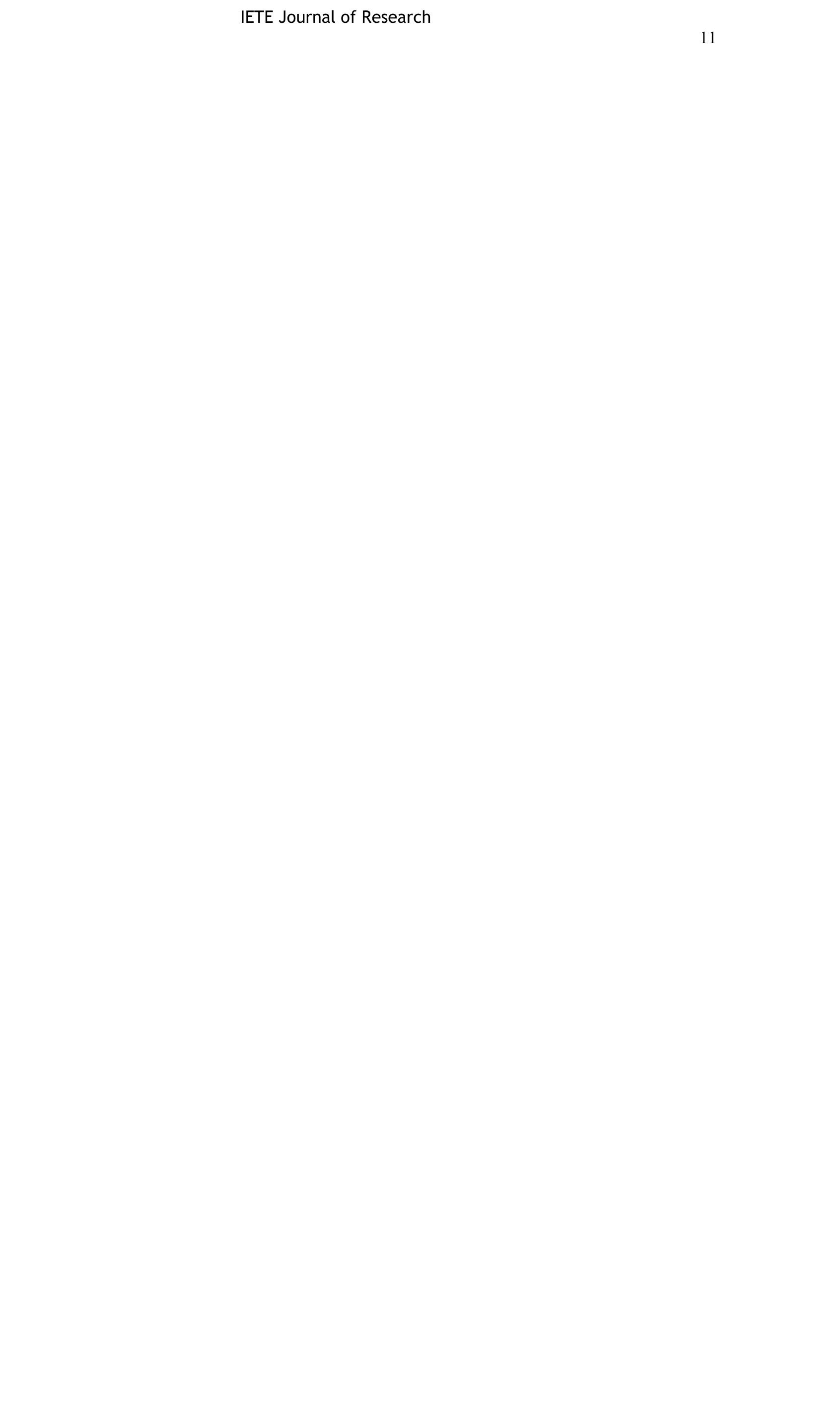
Table 1: Elective parameters of PSO for optimization of the WPD

\begin{tabular}{cccccc}
\hline $\boldsymbol{N}$ & $\boldsymbol{\theta}_{\max }$ & $\boldsymbol{\theta}_{\min }$ & $\boldsymbol{i}_{\max }$ & $\boldsymbol{c}_{\mathbf{1}}$ & $\boldsymbol{c}_{\mathbf{1}}$ \\
\hline 20 & 0.7 & 0.4 & 200 & 2 & 2 \\
\hline
\end{tabular}

Table 2: Designing parameters of PSO from several trials

\begin{tabular}{lccc}
\hline Trials & Target function values & $\theta_{1}$ & $\theta_{2}$ \\
\hline 18 & 0.99300 & 89.13 & 115.01 \\
\hline 26 & 0.78000 & 88.77 & 121.44 \\
\hline 35 & 0.75300 & 88.09 & 109.58 \\
\hline 57 & 0.50200 & 86.86 & 96.30 \\
\hline 79 & 0.11900 & 85.97 & 95.75 \\
\hline 86 & 0.00034 & 85.57 & 94.12 \\
\hline 158 & 0.00026 & 85.64 & 94.34 \\
\hline 186 & 0.00098 & 85.07 & 95.06 \\
\hline
\end{tabular}

Table 3: PSO design parameters

\begin{tabular}{cccccc}
\hline $\begin{array}{c}\boldsymbol{Y}_{\mathbf{1}} \\
(\mathbf{m h o})\end{array}$ & $\begin{array}{c}\boldsymbol{Y}_{\mathbf{2}} \\
(\mathbf{m h o})\end{array}$ & $\begin{array}{c}\boldsymbol{Z}_{\mathbf{1}} \\
(\mathbf{\Omega})\end{array}$ & $\begin{array}{c}\boldsymbol{Z}_{2} \\
(\boldsymbol{\Omega})\end{array}$ & $\begin{array}{c}\boldsymbol{\theta}_{\mathbf{1}} \\
(\mathbf{d e g} .)\end{array}$ & $\begin{array}{c}\boldsymbol{\theta}_{2} \\
(\mathbf{d e g})\end{array}$ \\
\hline $7.3 \times 10^{-3}$ & $6.83 \times 10^{-3}$ & 136.88 & 146.39 & 85.57 & 94.12 \\
\hline
\end{tabular}

Table 4: Dimensions of the DGS parameters in several cases

\begin{tabular}{ccccc}
\hline & Case1 & Case2 & Case3 & Case4 \\
\hline$L_{g 1}(\mathrm{~mm})$ & - & 10.80 & 10.80 & $\mathbf{1 0 . 8 0}$ \\
\hline$L_{g 2}(\mathrm{~mm})$ & 3.36 & 2.38 & 4.78 & $\mathbf{4 . 7 8}$ \\
\hline$L_{g 3}(\mathrm{~mm})$ & - & 7.70 & 7.70 & $\mathbf{7 . 7 0}$ \\
\hline$L_{g 4}(\mathrm{~mm})$ & - & - & 1.39 & $\mathbf{6 . 2 0}$ \\
\hline$W_{g 1}(\mathrm{~mm})$ & - & 1.39 & 3.26 & $\mathbf{1 . 3 9}$ \\
\hline$W_{g 2}(\mathrm{~mm})$ & 3.26 & 3.26 & - & $\mathbf{1 . 0 5}$ \\
\hline$W_{g 3}(\mathrm{~mm})$ & - & - & - & $\mathbf{0 . 3 4}$ \\
\hline$W_{g 4}(\mathrm{~mm})$ & - & - & 2 & $\mathbf{2}$ \\
\hline$g(\mathrm{~mm})$ & 2 & 2 & & \\
\hline
\end{tabular}


Table 5: Performance comparison of this work with other power divider

\begin{tabular}{c|ccccccc}
\hline Ref. & $\begin{array}{c}\text { Frequency } \\
(\mathbf{G H z})\end{array}$ & $\begin{array}{c}\text { Structure } \\
\text { topology }\end{array}$ & $\begin{array}{c}\mathbf{S}_{\mathbf{1 1}} \\
\mathbf{( d B})\end{array}$ & $\begin{array}{c}\mathbf{S}_{\mathbf{2 3}} \\
\mathbf{( d B )}\end{array}$ & $\begin{array}{c}\text { FBW } \\
(\mathbf{\%})\end{array}$ & $\begin{array}{c}\text { Circuit size } \\
\left(\mathbf{m m}^{\mathbf{2}}\right)\end{array}$ & $\begin{array}{c}\text { Circuit size } \\
\left(\lambda_{g} \times \lambda_{g}\right)\end{array}$ \\
\hline$[10]$ & $0.5-4.5$ & Triple-lines & $<-10$ & $<-13$ & 166 & 195.05 & $0.23 \times 0.08$ \\
{$[12]$} & $3.1-10.6$ & Microstrip & $<-10$ & $<-11$ & 117 & 517.5 & $0.56 \times 1.19$ \\
{$[13]$} & $2-5$ & Microstrip/slotline & $<-10$ & $<-13$ & 69 & 900 & $0.52 \times 0.52$ \\
{$[14]$} & $9.6-14.8$ & SIW & $<-10$ & $<-10$ & 42 & 2450 & $2.99 \times 1.49$ \\
{$[16]$} & $1-7$ & Microstrip/slotline & $<-10$ & $<-15$ & 141 & 2200 & $0.72 \times 1.08$ \\
This work & $\mathbf{0 . 8 - 4}$ & Dual-lines & $<-10$ & $<-10$ & $\mathbf{1 3 3}$ & $\mathbf{1 0 7 . 9 6}$ & $\mathbf{0 . 1 1} \times \mathbf{0 . 1 4}$ \\
\hline
\end{tabular}




\section{List of Figures}

Figure 1: The proposed structure of the WPD

Figure 2: Quarter-wavelength transmission-line and its equivalent parallel transmission-line structure

Figure 3: Resonance responses of a conventional quarter-wavelength transmission-line and the proposed PPTLs Figure 4: The convergence performance of the PSO algorithm

Figure 5: S-parameters and configuration of the proposed WPD

Figure 6: The DGS pattern etched on the ground-plane of the proposed WPD

Figure 7: The effect of DGS on the return-loss performance

Figure 8: Structure of the parallel transmission-lines in microstrip form. (a) Layout of the pair of parallel transmission-lines; (b) Parameters defining the dimensions of the upper transmission-line; (c) Parameters defining the dimensions of the lower transmission-line

Figure 9: Final configuration of the proposed WPD with DGS and the conventional WPD. (a) The proposed WPD; (b) The conventional WPD

Figure 10: The insertion-loss, return-loss and isolation performance of the proposed WPD with DGS and the conventional WPD

Figure 11: Photograph of the fabricated WPD. (a) Top layer; (b) Bottom layer

Figure 12: Measured S-parameters of the proposed WPD

Figure 13: Simulated and measured performance of the proposed WPD. (a) magnitude difference; (b) phase difference 


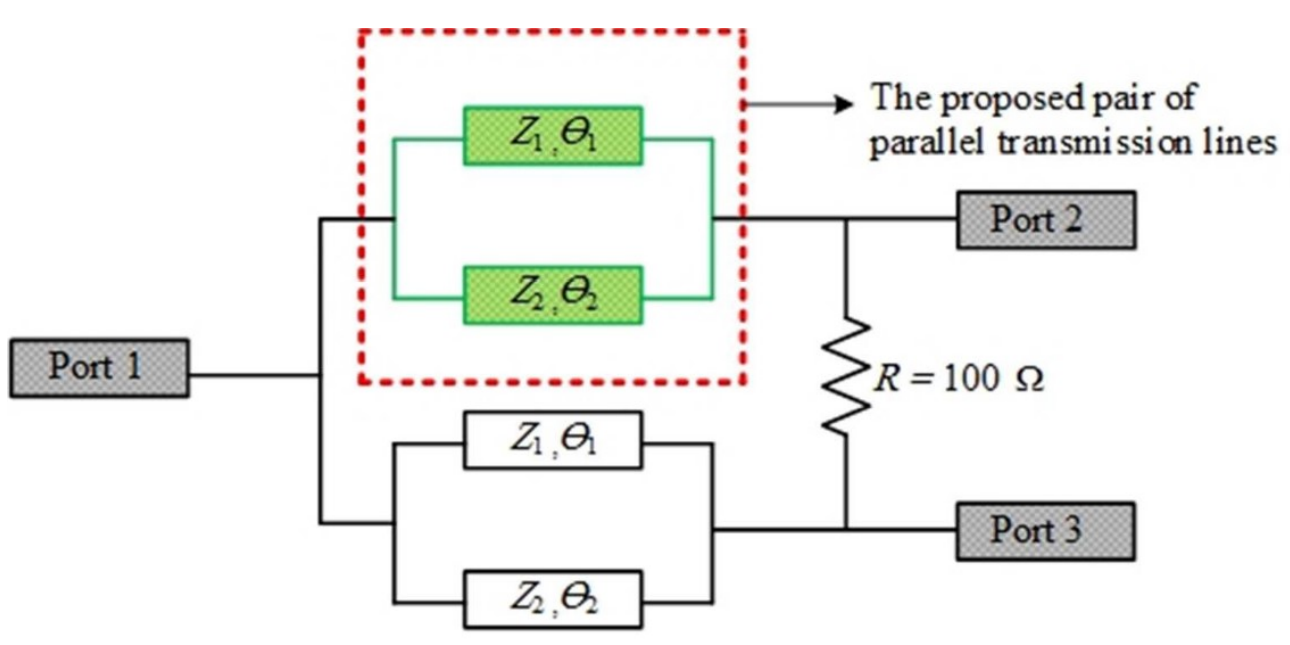

Figure 1: The proposed structure of the WPD $140 \times 68 \mathrm{~mm}(96 \times 96$ DPI) 


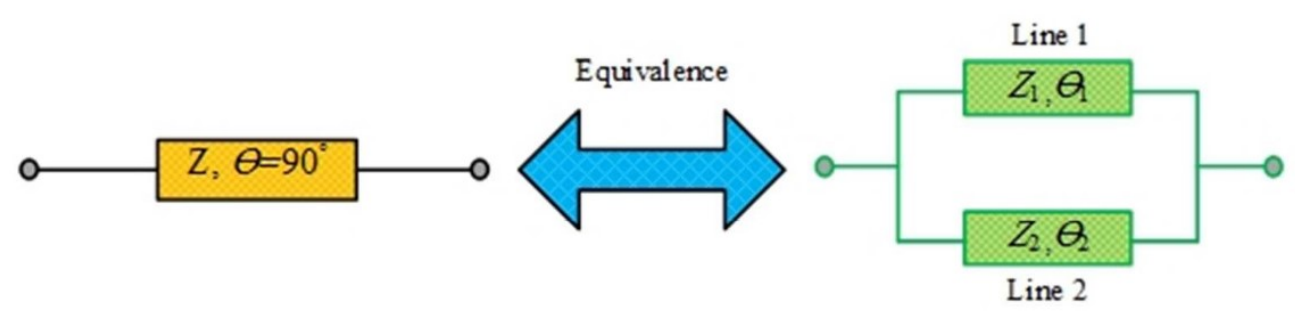

Figure 2: Quarter-wavelength transmission-line and its equivalent parallel transmission-line structure $147 \times 35 \mathrm{~mm}(96 \times 96 \mathrm{DPI})$ 


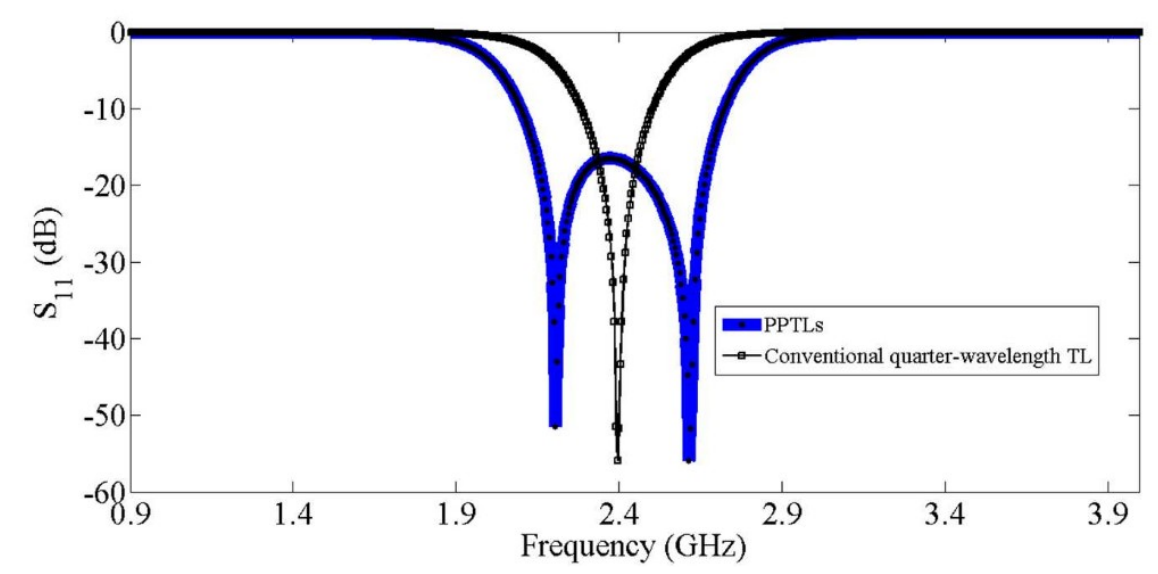

Figure 3: Resonance responses of a conventional guarter-wavelength transmission-line and the proposed $361 \times 159 \mathrm{~mm}(96 \times 96 \mathrm{DPI})$ 


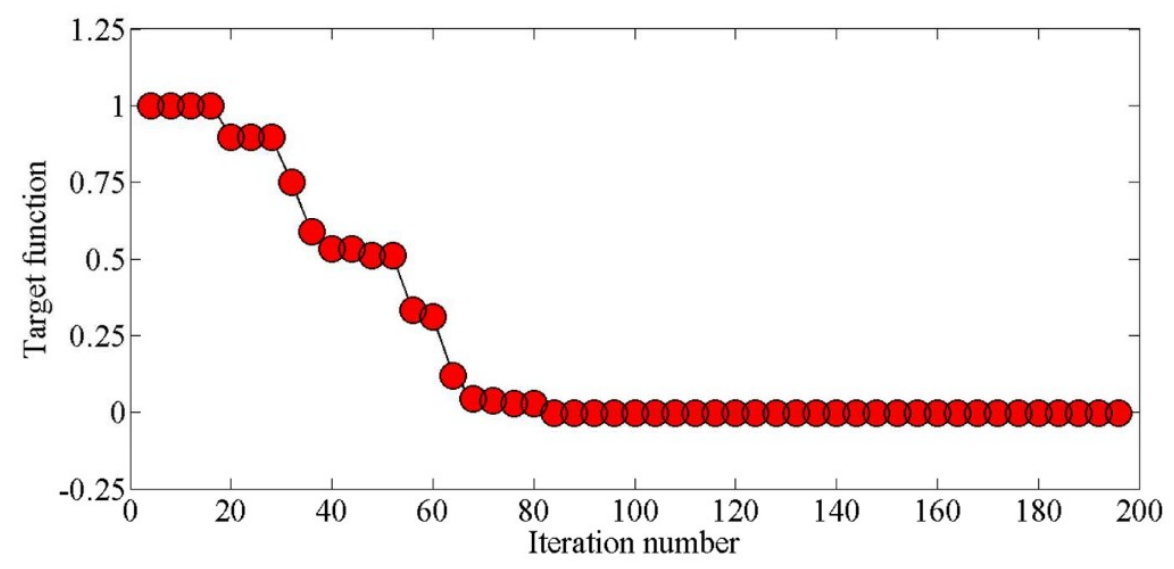

Figure 4: The convergence performance of the PSO algorithm $361 \times 159 \mathrm{~mm}(96 \times 96 \mathrm{DPI})$ 


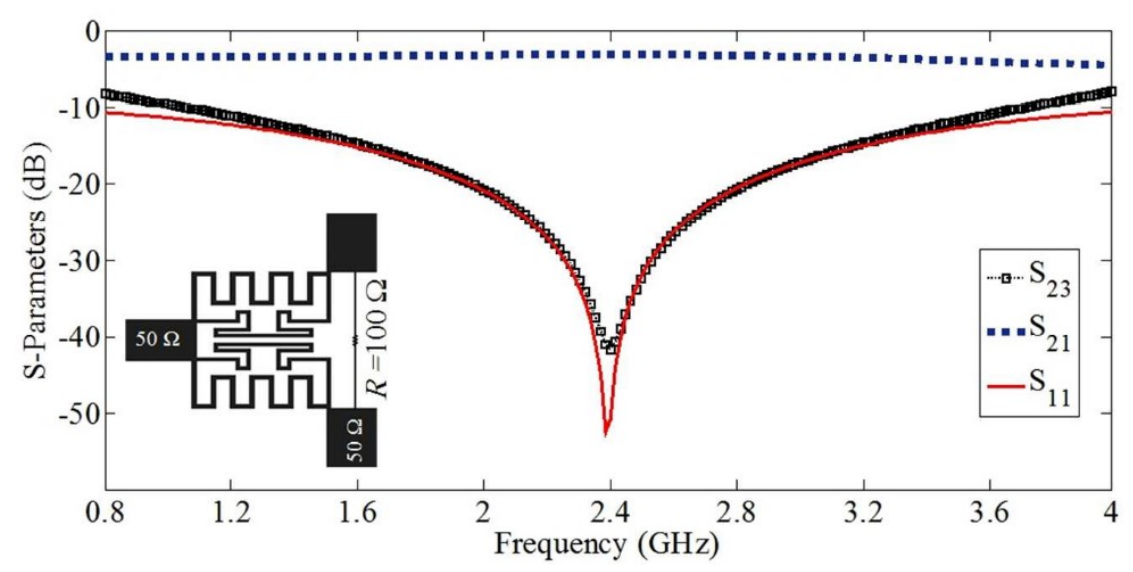

Figure 5: S-parameters and configuration of the proposed WPD $309 \times 136 \mathrm{~mm}(96 \times 96 \mathrm{DPI})$ 


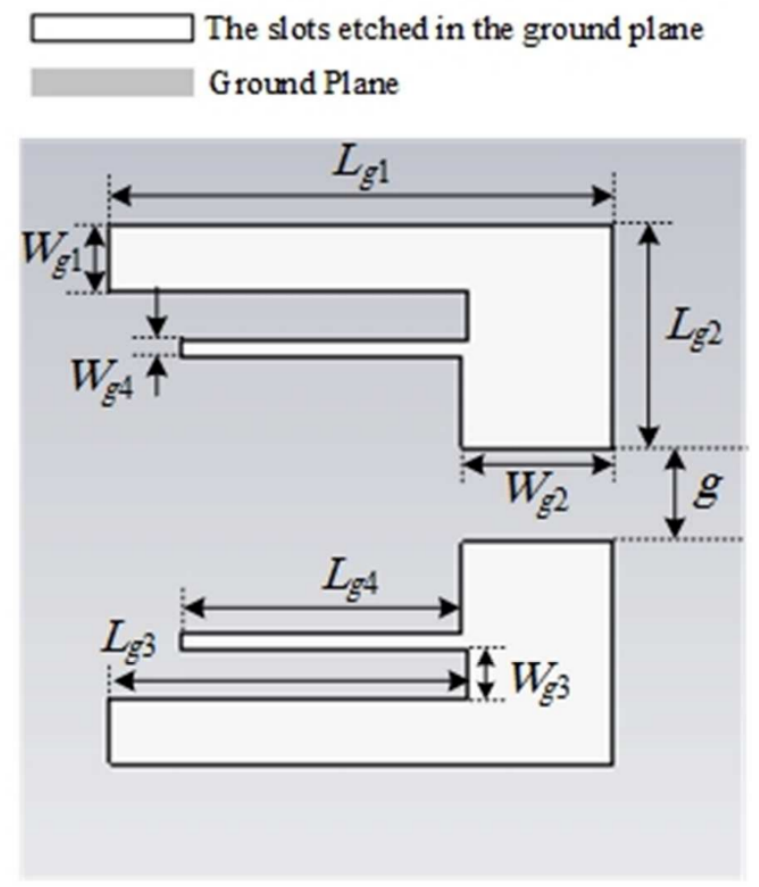

Figure 6: The DGS pattern etched on the ground-plane of the proposed WPD $77 \times 91 \mathrm{~mm}(96 \times 96$ DPI $)$ 


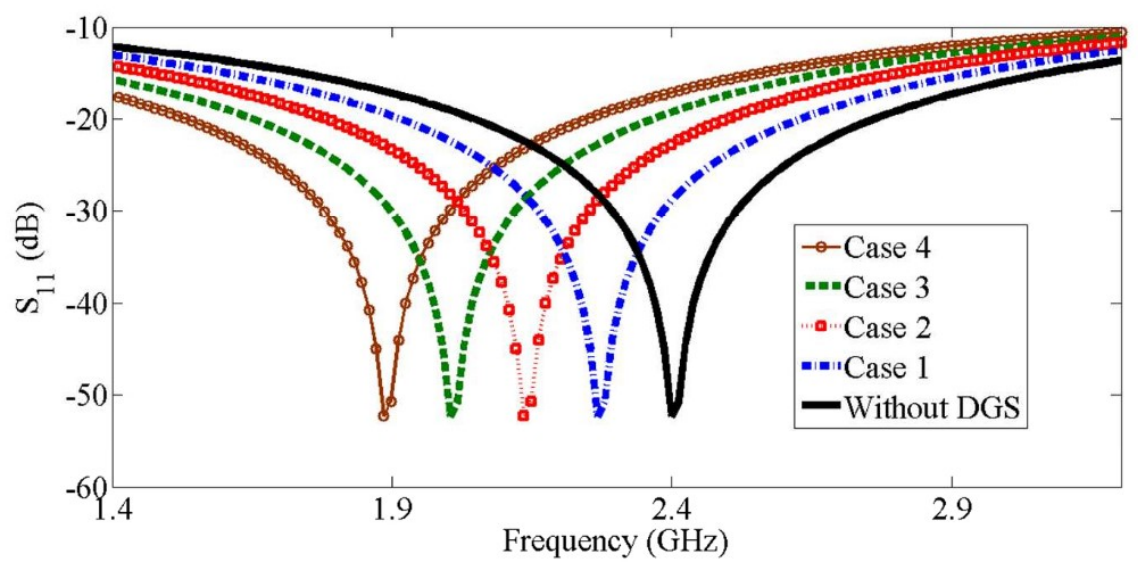

Figure 7: The effect of DGS on the return-loss performance $361 \times 159 \mathrm{~mm}(96 \times 96$ DPI) 


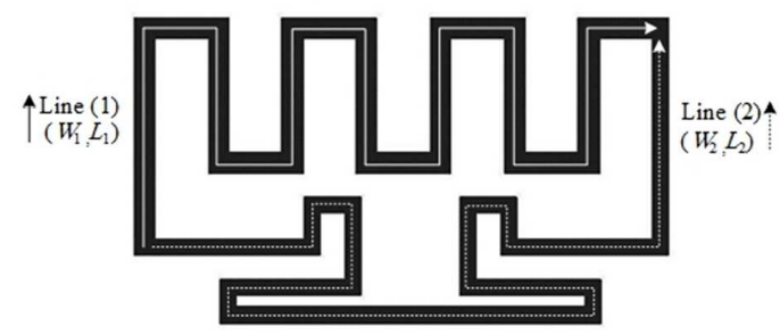

(a)

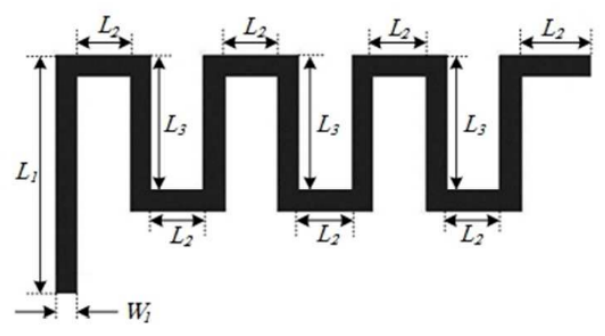

(b)

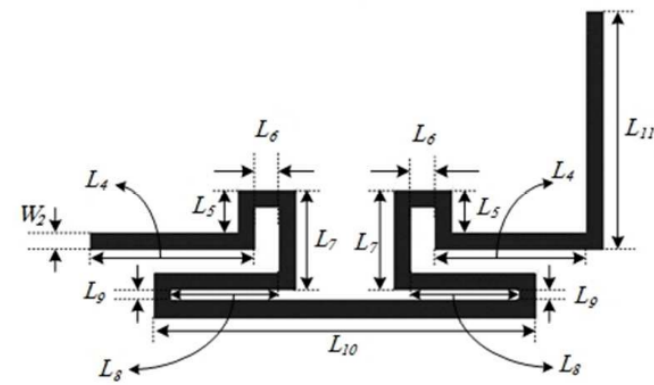

(c)

Figure 8: Structure of the parallel transmission-lines in microstrip form. (a) Layout of the pair of parallel transmission-lines; (b) Parameters defining the dimensions of the upper transmission-line; (c) Parameters defining the dimensions of the lower transmission-line 


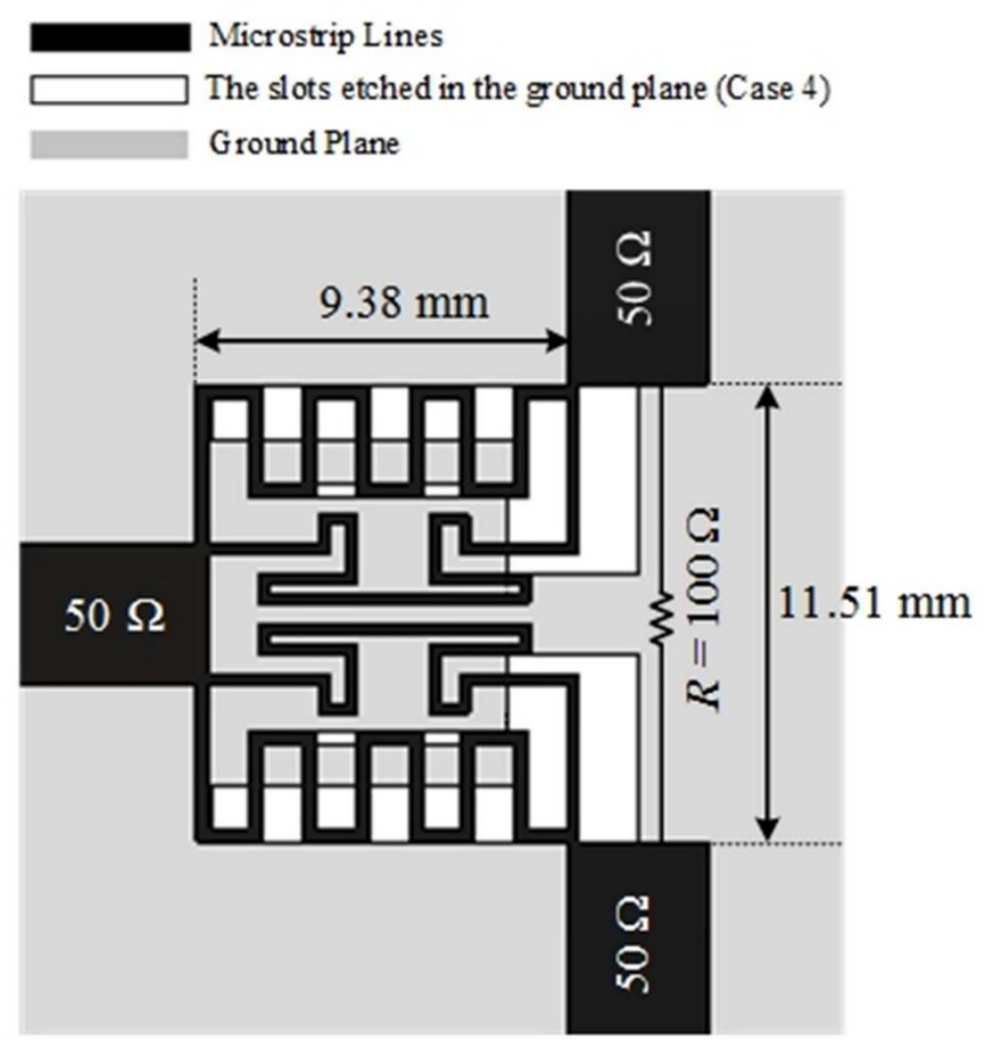

Figure 9: Final configuration of the proposed WPD with $\mathrm{DGS}$ and the conventional WPD. (a) The proposed $101 \times 105 \mathrm{~mm}(96 \times 96 \mathrm{DPI})$ 


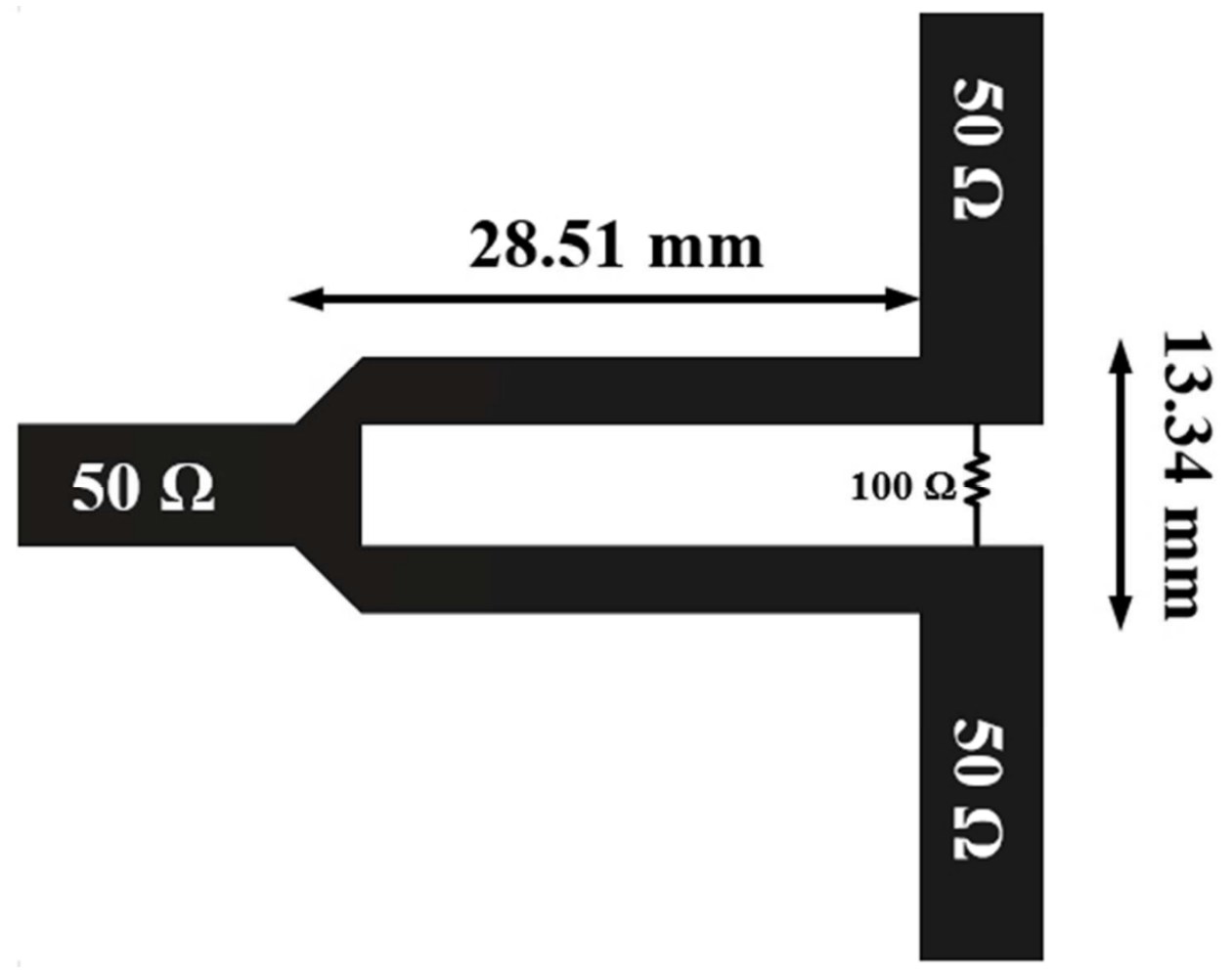

Figure 9: Final configuration of the proposed WPD with DGS and the conventional WPD. (b) The conventional WPD 


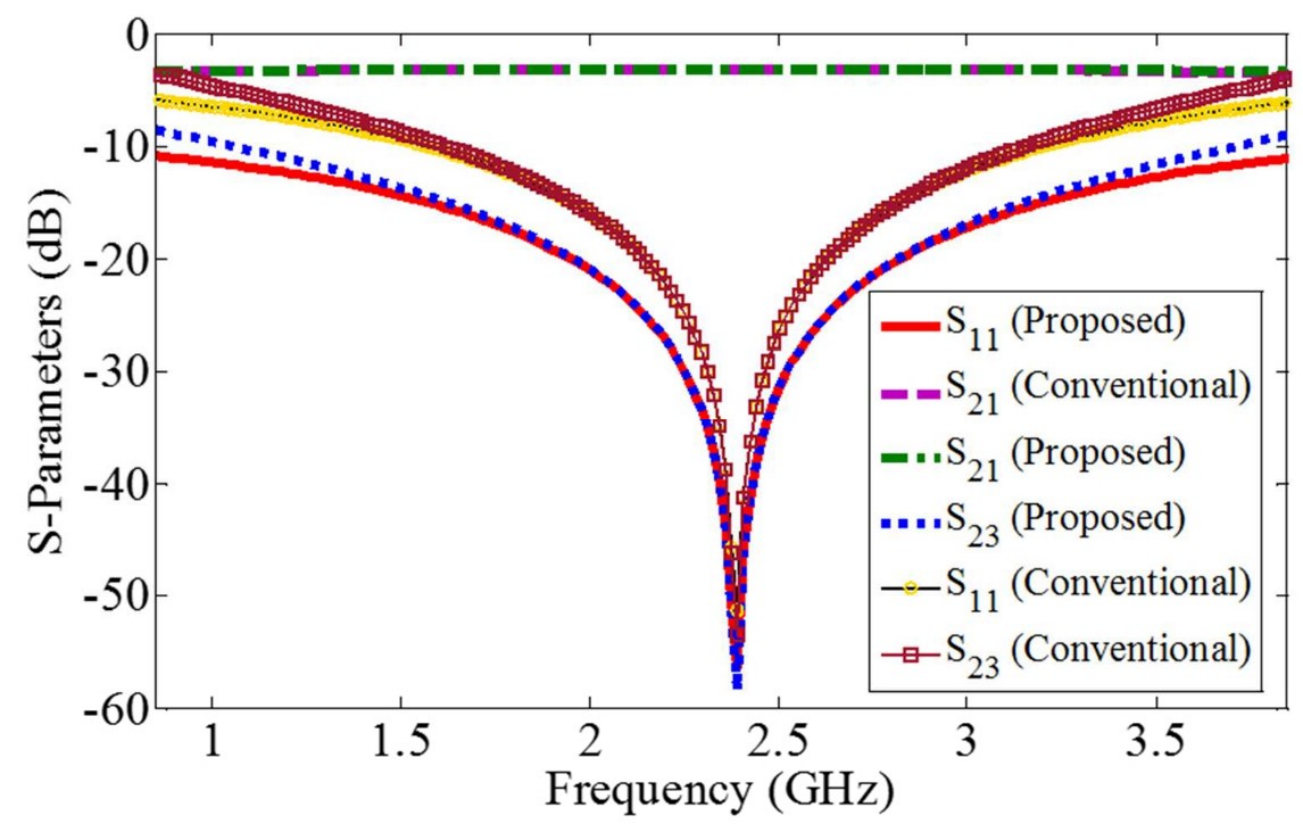

Figure 10: The insertion-loss, return-loss and isolation performance of the proposed WPD with DGS and the conventional WPD

$220 \times 140 \mathrm{~mm}(96 \times 96$ DPI $)$ 

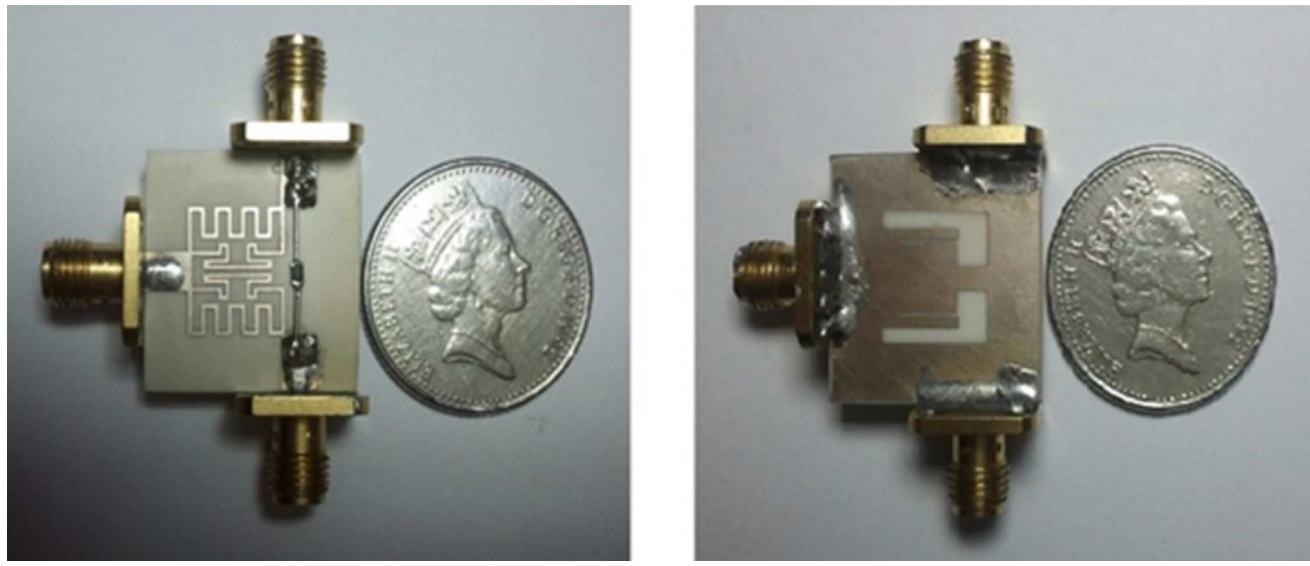

Figure 11: Photograph of the fabricated WPD. (a) Top layer; (b) Bottom layer $150 \times 64 \mathrm{~mm}(96 \times 96 \mathrm{DPI})$ 


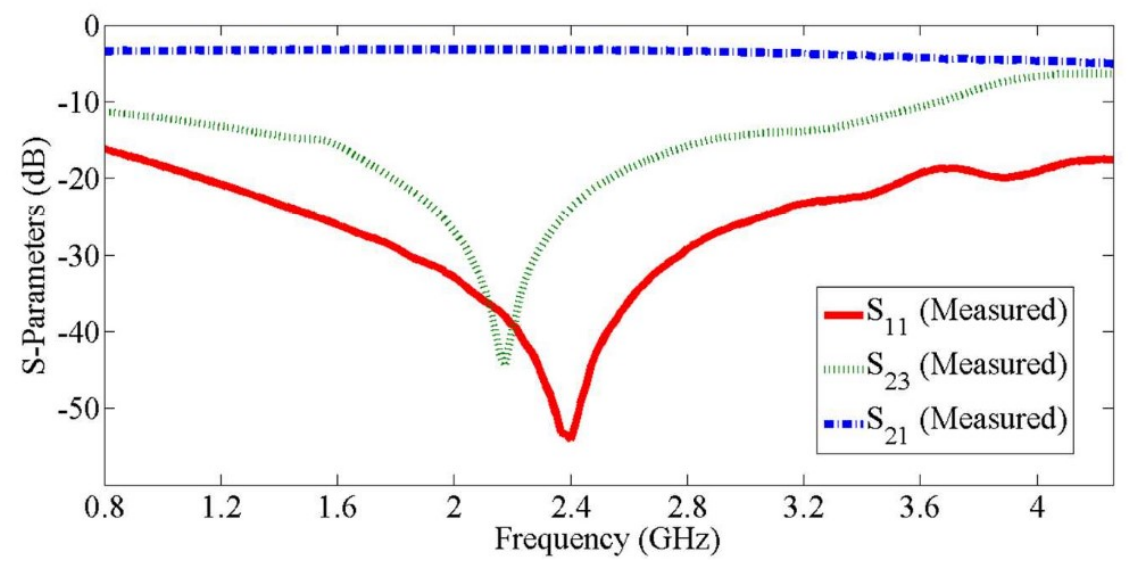

Figure 12: Measured S-parameters of the proposed WPD $361 \times 159 \mathrm{~mm}(96 \times 96$ DPI) 


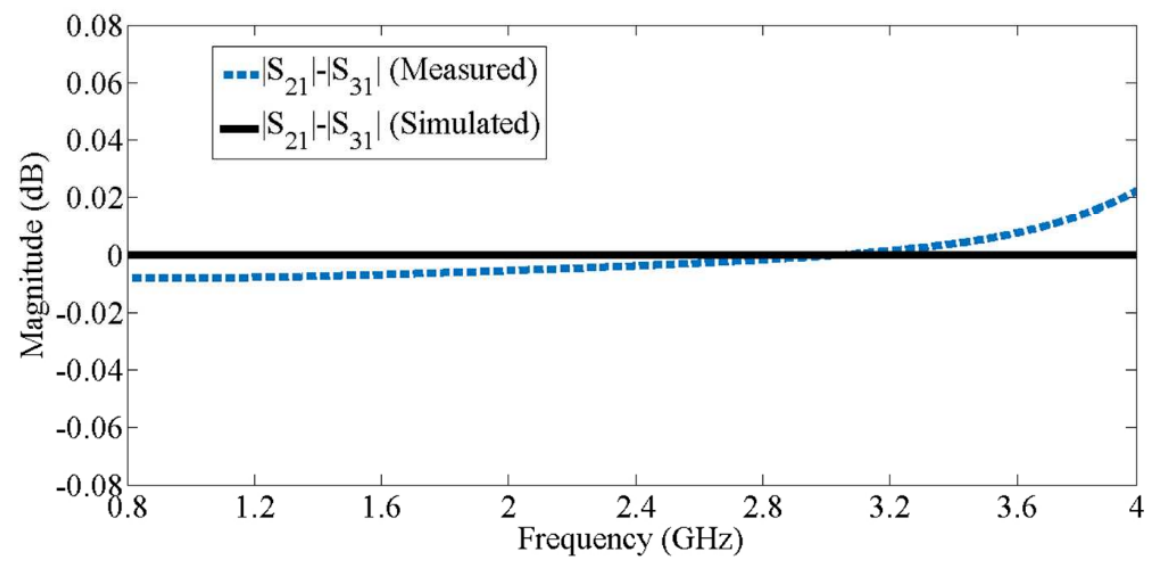

Figure 13: Simulated and measured performance of the proposed WPD. (a) magnitude difference; $361 \times 159 \mathrm{~mm}(96 \times 96 \mathrm{DPI})$ 


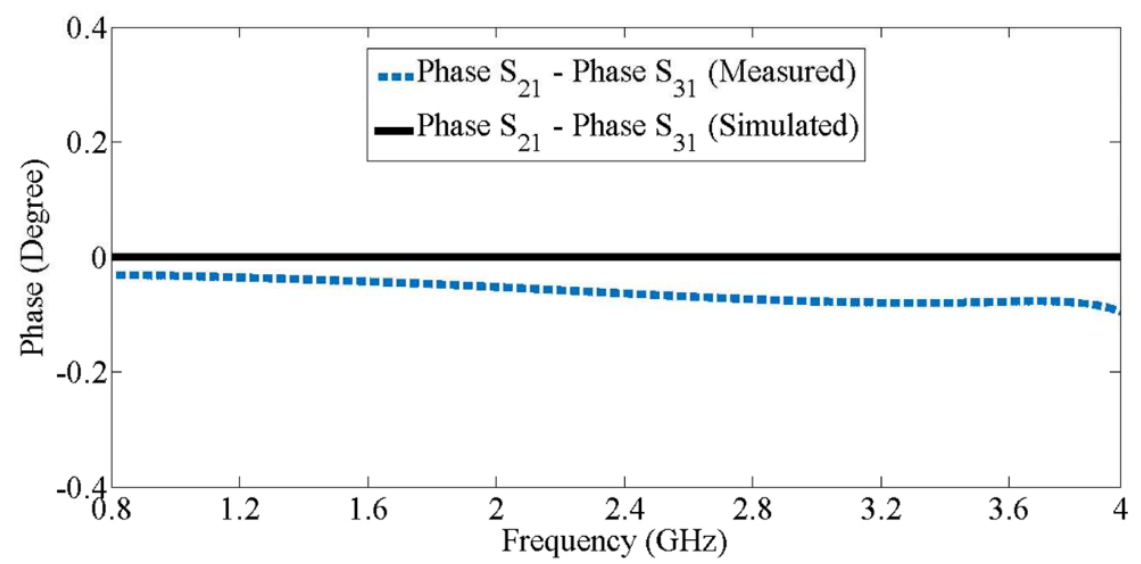

Figure 13: Simulated and measured performance of the proposed WPD. (b) phase difference $361 \times 159 \mathrm{~mm}$ (96 x 96 DPI) 